Supporting Information for:

\title{
NMR Studies of Ligand Binding to $\mathrm{P} 450_{\text {eryF }}$ Provides
}

\section{Insight into the Mechanism of Cooperativity}

Arthur G. Roberts ${ }^{a}$, M. Dolores Díaz ${ }^{a}$, Jed N. Lampe ${ }^{a}$, Laura M. Shireman ${ }^{a}$, Jeffrey S. Grinstead ${ }^{a}$,

Michael J. Dabrowski ${ }^{a}$, Josh T. Pearson ${ }^{a}$, Michael K. Bowman ${ }^{b}$, William M. Atkins ${ }^{a}$, A. Patricia

$$
\text { Campbell }{ }^{a, *}
$$

${ }^{a}$ Department of Medicinal Chemistry, School of Pharmacy, University of Washington, Box 357610, Seattle, WA 98195.

${ }^{b}$ Structural Biology and Microimaging, Battelle Northwest Laboratories, Richland, WA 99352-0999. 


\section{Footnotes}

${ }^{1} \% \Delta \mathrm{I}=\Delta \mathrm{I} / \mathrm{I}^{\text {free }} \times 100$, where $\Delta \mathrm{I}=\mathrm{I}^{\text {free }}-\mathrm{I}^{\text {bound }}$, and where $\mathrm{I}^{\text {free }}$ is the intensity at 9-AP:P450 $0_{\text {eryF }}=0$, and $\mathrm{I}^{\text {bound }}$ is the intensity at 9-AP:P450 $0_{\text {ery }}=2.0$.

${ }^{2} \mathrm{P} 5$ and $\mathrm{P} 8$ are comprised of two overlapping resonances, so intensity measurements reflect a weighted average. ${ }^{3}\left(\Delta \delta_{\mathrm{av}}=\left[\Delta \delta_{\mathrm{NH}}{ }^{2}+\left(\Delta \delta_{15 \mathrm{~N}} / 5\right)^{2}\right]^{1 / 2}\right.$, where $\Delta \delta=\delta^{\text {free }}-\delta^{\text {bound }}$, (see references 34, 35 of the manuscript) and where $\delta^{\text {free }}$ is the chemical shift at TST:P450 eryF $=0$, and $\delta^{\text {bound }}$ is the chemical shift at TST:P450 eryF $=12.0$.

${ }^{4} \mathrm{P} 5$ is comprised of at least two overlapping resonances that shift independently of each other in the presence of TST. The peak positions for both resonances are given in the table at the start and end of the titration.

${ }^{5} \mathrm{P} 8$ is comprised of at least two overlapping resonances that shift independently of each other in the presence of TST. The peak positions for the most shifted of the two resonances are given in the table for all stages of the titration.

${ }^{6} \mathrm{P} 12$ was not observed at TST:P450 eryF $=12$, so $\Delta \delta_{\mathrm{av}}$ was taken from TST:P450 eryF $=4.4$ (value given in brackets). 


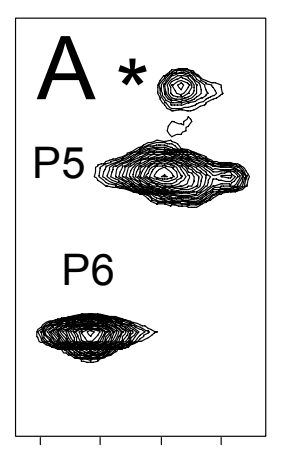

$\begin{array}{llllll}8.4 & 8.3 & 8.2 & 8.1\end{array}$

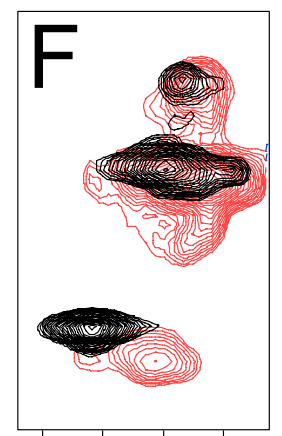

$\begin{array}{llll}8.4 & 8.3 & 8.2 & 8.1\end{array}$

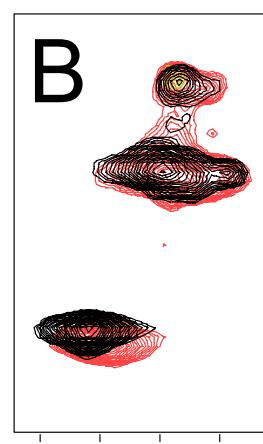

$\begin{array}{lllll}8.4 & 8.3 & 8.2 & 8.1\end{array}$

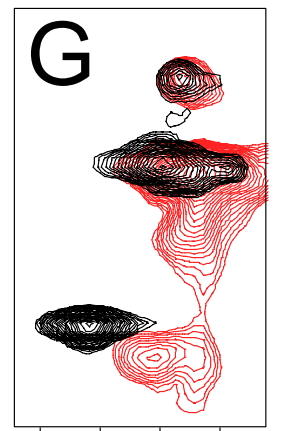

$\begin{array}{llll}8.4 & 8.3 & 8.2 & 8.1\end{array}$
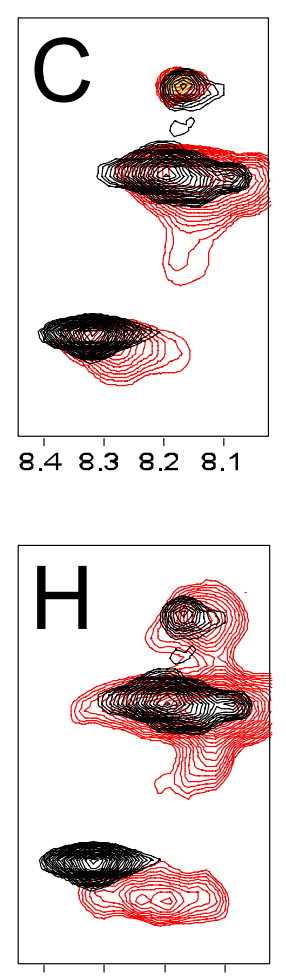

$\begin{array}{llll}8.4 & 8.3 & 8.2 & 8.1\end{array}$

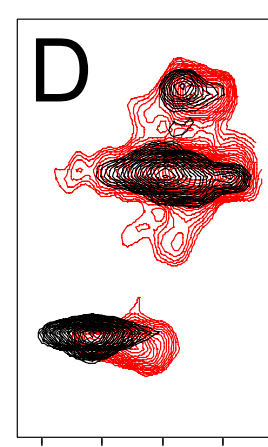

$\begin{array}{llll}8.4 & 8.3 & 8.2 & 8.1\end{array}$

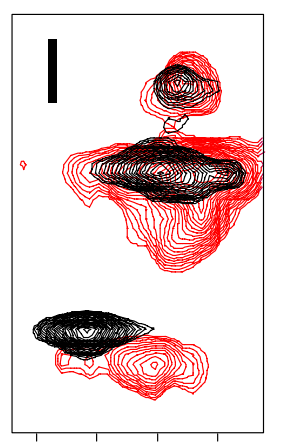

$8.48 .3 \quad 8.2 \quad 8.1$
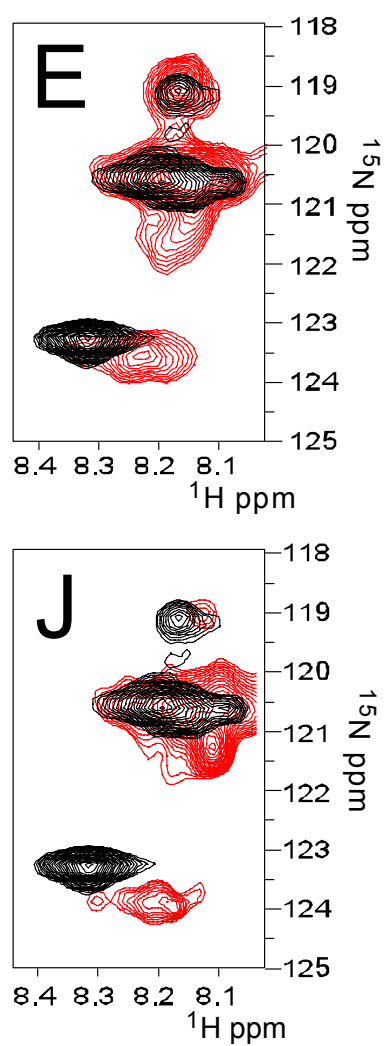

Figure S1. $2 D-\left\{{ }^{15} N,{ }^{l} H\right\}-H S Q C$ spectra expansions showing the complex spectral behavior exhibited by the P5 and P6 signals. The P5 and P6 signals of (a) ligand-free ${ }^{15} \mathrm{~N}-\mathrm{Phe}$ labeled P450 eryF (black) and with (b) 0.5 , (c) 1.1 , (d) 1.8 , (e) 2.5 , (f) 3.1, (g) 3.8, (h) 4.4, (i) 6.0, and (j) 12 molar equivalents of TST. 


\begin{tabular}{|c|c|c|c|c|c|c|c|c|}
\hline & \multicolumn{8}{|c|}{ 9-AP:CYP ${ }_{\text {eryF }}$ (molar equivalents) } \\
\hline Peak \# & $\mathbf{0 . 0}$ & 0.8 & 1.1 & 1.2 & 1.4 & 1.6 & 2.0 & $\% \Delta I^{I}$ \\
\hline P1 & 5.3 & 4.0 & 3.5 & 2.4 & 1.4 & 2.0 & 1.6 & 70 \\
\hline $\mathbf{P 2}$ & 6.1 & 3.5 & 3.0 & 3.0 & 1.9 & 1.4 & 0 & 100 \\
\hline $\mathbf{P 3}$ & 5.5 & 3.0 & 4.4 & 3.8 & 2.6 & 1.9 & 2.0 & 64 \\
\hline $\mathbf{P 4}$ & 3.4 & 2.0 & 1.9 & 3.4 & 2.0 & 1.2 & 2.0 & 41 \\
\hline $\mathbf{P 5}^{2}$ & 8.6 & 6.0 & 4.7 & 4.4 & 2.9 & 2.6 & 2.0 & 77 \\
\hline P6 & 6.3 & 2.6 & 2.2 & 2.4 & 1.4 & 0.7 & 0 & 100 \\
\hline P7 & 4.5 & 4.0 & 3.5 & 3.0 & 3.7 & 3.7 & 2.8 & 38 \\
\hline $\mathbf{P 8}^{2}$ & 8.3 & 6.0 & 6.0 & 5.6 & 4.5 & 4.8 & 4.0 & 52 \\
\hline P9 & 6.3 & 3.5 & 3.1 & 2.2 & 1.9 & 1.6 & 0.1 & 98 \\
\hline $\mathbf{P 1 0}$ & 6.3 & 5.2 & 5.5 & 4.6 & 4.4 & 4.1 & 4.1 & 35 \\
\hline P11 & 8.4 & 7.6 & 6.9 & 7.6 & 6.0 & 6.8 & 6.1 & 27 \\
\hline P12 & 3.2 & 2.3 & 2.4 & 1.2 & 0.9 & 0.5 & 0 & 100 \\
\hline P13 & 3.4 & 2.0 & 2.4 & 2.6 & 2.3 & 2.3 & 2.2 & 35 \\
\hline P14 & 9.6 & 7.9 & 9.8 & 8.2 & 7.3 & 8.1 & 8.0 & 17 \\
\hline
\end{tabular}

Table S1. 2D- $\left\{{ }^{1} \mathrm{H}^{15} \mathrm{~N}\right\}$-HSQC peak intensities measured for $200 \mu \mathrm{M}\left[{ }^{15} \mathrm{~N}-\mathrm{Phe}\right]-\mathrm{P} 450_{\text {eryF }}$ in the presence of 9-AP. 


\begin{tabular}{|c|c|c|c|c|c|c|c|c|c|c|c|}
\hline & \multicolumn{11}{|c|}{ TST: CYP ${ }_{\text {eryF }}$ (molar equivalents) } \\
\hline Peak\# & $\mathbf{0 . 0}$ & 0.5 & 1.1 & $\mathbf{1 . 8}$ & 2.5 & 3.1 & 3.8 & 4.4 & 6.0 & $\mathbf{1 2 . 0}$ & $\Delta \delta_{\mathrm{av}}^{3}$ \\
\hline \multirow{2}{*}{$\begin{array}{r}\mathbf{P 1}\left(\delta^{1} \mathbf{H}\right) \\
\left(\delta^{15} \mathbf{N}\right)\end{array}$} & 8.93 & 8.96 & 8.96 & 8.97 & 8.99 & 8.99 & 8.98 & 9.00 & 9.00 & 9.00 & 0.08 \\
\hline & 117.4 & 117.4 & 117.4 & 117.3 & 117.4 & 117.4 & 117.6 & 117.6 & 117.6 & 117.6 & \\
\hline \multirow[t]{2}{*}{$\mathbf{P 2}$} & 8.98 & 8.97 & 8.97 & 8.94 & 8.95 & 8.94 & 8.94 & 8.93 & 8.93 & 8.93 & 0.13 \\
\hline & 123.3 & 123.2 & 123.0 & 122.8 & 122.7 & 122.7 & 122.8 & 122.8 & 122.7 & 122.7 & \\
\hline \multirow[t]{2}{*}{$\mathbf{P 3}$} & 8.89 & 8.90 & 8.90 & 8.91 & 8.94 & 8.92 & 8.92 & 8.89 & 8.91 & 8.91 & 0.03 \\
\hline & 126.3 & 126.3 & 126.2 & 126.2 & 126.2 & 126.1 & 126.3 & 126.3 & 126.2 & 126.2 & \\
\hline \multirow[t]{2}{*}{$\mathbf{P 4}$} & 8.46 & 8.45 & 8.45 & 8.43 & 8.45 & 8.43 & 8.44 & 8.43 & 8.43 & 8.43 & 0.03 \\
\hline & 131.6 & 131.4 & 131.4 & 131.5 & 131.6 & 131.4 & 131.7 & 131.7 & 131.6 & 131.6 & \\
\hline \multirow[t]{2}{*}{ P5 (res. 1) } & 8.20 & - & - & - & - & - & - & - & - & 8.10 & 0.17 \\
\hline & 120.6 & - & - & - & - & - & - & - & - & 121.3 & \\
\hline \multirow[t]{2}{*}{ P5 (res. 2) } & 8.20 & - & - & - & - & - & - & - & - & 8.05 & 0.15 \\
\hline & 120.6 & - & - & - & - & - & - & - & - & 120.7 & \\
\hline \multirow[t]{2}{*}{ P6 } & 8.33 & 8.28 & 8.27 & 8.25 & 8.23 & 8.22 & 8.21 & 8.20 & 8.20 & 8.20 & 0.18 \\
\hline & 123.3 & 123.5 & 123.6 & 123.5 & 123.6 & 123.8 & 123.8 & 123.9 & 123.8 & 123.9 & \\
\hline \multirow[t]{2}{*}{$\mathbf{P 7}$} & 7.94 & 7.96 & 7.97 & 7.97 & 7.97 & 7.99 & 8.00 & 7.97 & 7.99 & 7.99 & 0.08 \\
\hline & 111.9 & 112.2 & 112.0 & 112.1 & 112.1 & 112.3 & 112.3 & 112.3 & 112.1 & 112.2 & \\
\hline \multirow[t]{2}{*}{$\mathbf{P 8}^{5}$} & 7.93 & 7.93 & 7.93 & 7.92 & 7.91 & 7.93 & 7.92 & 7.91 & 7.92 & 7.92 & 0.02 \\
\hline & 117.8 & 117.8 & 117.7 & 117.7 & 117.8 & 117.8 & 117.9 & 118.0 & 117.8 & 117.9 & \\
\hline \multirow[t]{2}{*}{ P9 } & 7.81 & 7.83 & 7.85 & 7.88 & 7.89 & 7.89 & 7.92 & 7.89 & 7.93 & 7.93 & 0.12 \\
\hline & 120.0 & 120.0 & 119.9 & 120.1 & 120.1 & 120.1 & 120.1 & 120.2 & 120.0 & 120.1 & \\
\hline \multirow[t]{2}{*}{ P10 } & 7.57 & 7.58 & 7.58 & 7.57 & 7.58 & 7.59 & 7.59 & 7.57 & 7.59 & 7.59 & 0.03 \\
\hline & 116.4 & 116.4 & 116.3 & 116.3 & 116.3 & 116.4 & 116.4 & 116.5 & 116.3 & 116.3 & \\
\hline \multirow[t]{2}{*}{ P11 } & 7.29 & 7.28 & 7.28 & 7.26 & 7.27 & 7.28 & 7.28 & 7.26 & 7.27 & 7.27 & 0.03 \\
\hline & 116.6 & 116.7 & 116.6 & 116.6 & 116.7 & 116.7 & 116.7 & 116.8 & 116.6 & 116.7 & \\
\hline \multirow[t]{2}{*}{$\mathbf{P 1 2}^{6}$} & 7.20 & 7.22 & 7.23 & 7.25 & 7.26 & 7.23 & 7.24 & 7.24 & - & - & 0.13 \\
\hline & 114.1 & 114.2 & 114.2 & 114.3 & 114.4 & 114.3 & 114.3 & 114.7 & - & - & \\
\hline \multirow[t]{2}{*}{$\overline{\text { P13 }}$} & 7.15 & 7.16 & 7.17 & 7.22 & 7.23 & 7.24 & 7.26 & 7.23 & 7.23 & 7.24 & 0.10 \\
\hline & 118.2 & 118.3 & 118.2 & 118.2 & 118.3 & 118.3 & 118.3 & 118.4 & 118.3 & 118.4 & \\
\hline \multirow[t]{2}{*}{ P14 } & 6.97 & 6.98 & 6.98 & 6.98 & 6.98 & 6.99 & 6.99 & 6.97 & 6.98 & 6.98 & 0.02 \\
\hline & 114.7 & 114.8 & 114.7 & 114.7 & 114.8 & 114.9 & 114.9 & 115.2 & 114.9 & 114.9 & \\
\hline
\end{tabular}

Table S2. ${ }^{1} \mathrm{H}$ and ${ }^{15} \mathrm{~N}$ chemical shifts of the $2 \mathrm{D}-\left\{{ }^{1} \mathrm{H}-{ }^{15} \mathrm{~N}\right\}-\mathrm{HSQC}$ measured for $280 \mu \mathrm{M}\left[{ }^{15} \mathrm{~N}\right.$-Phe]$\mathrm{P} 450 \mathrm{eryF}$ in the presence of TST. 